\title{
A Mini Review on Tamarind (Tamrindus indica L.) for Induction of Ornamental Phenotype and Seedlessness
}

\section{Christopher J Catanzaro ${ }^{4}$, Kameswari PL $^{2}$, Sujan Mamidi ${ }^{3}$, John A Parry ${ }^{5}$, Mohammad J Iqbal ${ }^{3}$, Anwar A Hamama Harbans L Bhardwaj ${ }^{5}$, Bhavani SVP ${ }^{1}$ and Satya SS Narina ${ }^{4 *}$}

\author{
${ }^{1}$ Andhra Pradesh Agricultural University, Rajahmundry, Andhra Pradesh, India \\ ${ }^{2}$ Andhra Pradesh Horticultural University, V. R. Gudem, Andhra Pradesh, India \\ ${ }^{3}$ Department of Plant Sciences, North Dakota State University, USA \\ ${ }^{4}$ Department of Agriculture and Human Ecology, Virginia State University, Petersburg, USA \\ ${ }^{5}$ Agricultural Research Station, Department of Agriculture, Virginia State University, Petersburg, USA \\ *Corresponding Author: Satya SS Narina, Department of Agriculture, Virginia State University, Petersburg, USA.
}

Received: September 25, 2019; Published: October 14, 2019

DOI: 10.31080/ASNH.2019.03.0505

\begin{abstract}
Consumption of fresh, unprocessed ripe fruits, nuts or salad vegetables is an essential component of healthy diet. Many above ground plant part of tamarind are edible and highly nutritious and have many industrial use values. Cytoplasmic male sterility (CMS) for production of cybrids, and self incompatibility (SI) are key to produce seedlessness in tamarind fruits, as is the application of growth regulating hormones (GRH) during pre-anthesis stage. Mutation breeding is the most viable breeding methodology to induce polyploidy and for development of cultivars with ornamental use. Tamarind cultivars with purely green foliage type, seedless fruiting type, and non-fruiting ornamental types would benefit food and horticultural industries. Development of morphological descriptors with molecular markers associated with important fruit traits, would assist with future research and strengthen germplasm resources. Keywords: Tissue Culture; Mutation; CMS; Growth Regulators; Tropical; Fruit Vegetable.
\end{abstract}

\section{Introduction}

Tamarind (Tamarindus indica L.,) is a tropical tree in the family Fabaceae (Leguminosae: Caesalpinioideae) whose commercial food products are used widely around the world. Mature trees are drought tolerant and have deep taproot system, wide crown spreading with abundant, evergreen foliage. Vegetative growth, flowering and fruiting depend on water availability. Trees typically produce two crops of fruit per year, fresh vegetative greens throughout the year in India [1-3]. Because tamarind tree produces fruits of table fruit value and vegetable use, it is considered as fruit-vegetable.

The consumption of tamarind leaf is very rarely seen in modern days where less nutritional spinach (provides Fe and $\mathrm{Zn}$ ) replaced most of the leafy greens by its availability at the cheapest price and easy adaptability to grow in all climatic conditions globally. The leaves of tamarind provides ascorbic acid that plays major role in absorption of metabolites and many minerals and vitamins for health and nutrition. The presence of calcium, iron and phosphorous is one of the unique features that plays role in generation of antibodies by being major constituents of photochemical energy that is required for human health in providing antioxidants from fresh green leaf salad and green fruit, while fruit pulp has many uses in medicine and food industry. Seed kernel and ripened sweet fruits are a good snack with excellent nutritive values providing carbohydrates, proteins, essential vitamins and minerals [2,3].

Tamarind seeds are a relatively new, cheap, and underutilized source of starch in food applications [4]. Additional products are obtained from tamarind's fresh green immature fruits, mature and ripened fruits, fruit pulp, seeds, leaves, flowers and their biproducts, pulp from tree, seed coats or hulls, wood and its biproducts, and firewood and charcoal from tree trunks and branches. These products have industrial uses related to food, pharmaceuticals, paper and furniture, and forestry $[2,3]$.

Perennial trees like palm species of tropical origin have been widely adopted for landscape use in warmer climates due to their ornamental traits. Southern states in the US (for example in Texas, Florida, California and Arizona) have subtropical zones where most palms are planted in rows or in groups along highways. The new species of tamarind, could be used along roadways where other palms like neredu pandu or malabar plum or black plum or java plum (Syzygium cumini L., Myrtaceae), tati pandu or toddy palm or 
palmyra palm (Borassus flabellifer L., Arecaceae), and eetha pandu or date palm (Phoenix dactylifera L., Arecaceae) including coconut (Cocos nucifera L., Arecaceae) are grown or inside the gardens or state parks to conserve germplasm, enhance biodiversity and aesthetics with their beautiful green fronds, colorful fruit and flower bunches.

Tamarind has bisexual, five-petaled yellow flowers of $2.5 \mathrm{~cm}$ wide, attracttive with orange or red streaks borne in racemes. Tamarind species can also provide many of the same ecological services of other trees, with an added benefit of nitrogen fixation. Purification of constantly polluting air and soil with increasing population, industries by healthy breeze from its dense foliage, beautiful flower bunches, might potentially replace some of the trees with low ecological value in areas wherever there is a possibility to grow this healthy and nutritious tree species.

Some parts of USA are enjoying the fresh coconut water of its own land in its natural form, so many fruits from different species of palm trees or other non native fruit trees by the sincere efforts of eminent scientists, international scientific faculty, private or public industries, and growers. The major fruit vegetables, where the crop is used both as fruit and vegetable use for food preparations as well as with extreme potential for ornamental and industrial crop use are cashew (Anacardium occidentale, Anacardiaceae), coconut, guava (Psidium gujava, L., Myrtaceae), jackfruit (Artocarpus integrefolia, Moraceae), mango (Mangifera indica, L., Anacardiaceae) , papaya (Carica papaya, Caricaceae), and tamarind. These crops have national economic, food and ornamental use values.

While plant breeder's have improved many multiseeded fruit crops including custard apple and pomegranate, seedlessness has been induced in certain table grapes, citrus and watermelons for large scale commercial production. Growth regulators, parthenocarpy, male sterility, self-incompatibility and chromosomal manipulations are the primary factors contributed for seedlessness in fruits.

Considering the fact that a number of tropical perennial fruit crops have been successfully introduced in the United States (US), potential long term and wider use of tamarind fruit pulp and its products due to its values in health and nutrition, authors like to reveal potential areas for tamarind crop improvement for seedless fruit production. The attempts in crop improvement for seedlessness benefit not only for its table purpose fruit and pulp uses, but also for its wider adaptation as an ornamental perennial tree.

Why there is a need for new crop introduction?

Challenges exist regarding protection of tamarind germplasm in a changing world. These issues include loss of accessions due to rapid deforestation (Eg., southern Malwai) and urbanization, lack of documentation of accessions, lack of accessibility to remote locations, genetic erosion due to use of elite trees for timber and fuelwood [5]. Other main reason could also be due to frequent transfer of sincere research personal, changes in political parties, no accessibility to digital resources or low cost germplasm conservatory storage options due to remote locations of growing regions as well as no access to researchers in those remote research stations to publish the details of the generated data pertaining to important breeding traits of both food and economic value, which might have lead to eventual loss of data as well as some elite germplasm resources of various crops. Collection of tamarind germplasm as clonal selections, grafts or seeds for long-term conservation by planting collections in parks, in research stations, arboreta, or on private property) at various locations globally would ensure protection of this valuable plant germplasm for future generations.

Although many perennial tress with edible or non-edible fruits were useful in many ways for human life and living till today, they are not benefitted from improved and innovative technology we had today as necessary for their cultivar improvement. For example, coconut (water and copra), and few date palm trees (fibrous fruit pulp from mesocarp, tender roots, copra from nuts) were used for foods rich in carbhohydrates, invert sugars (glusoce and fructose) fibre, iron, potassium and vitamins. Sapindus (non-edible fruit, perennial tree), an economically important tree genera where the fruit and nut are processed for soapnut powder, and used in most shampoos we use today. In coconut (edible fruit, perennial tree), the fibrous mesocarp is used for making ropes and so many industrial products. The dehulled seed is economically valued costly nut in some perennial trees (cashew, coconut). But in most fruit trees, the seed (nut) is used only for raising seedlings for grafts or left unused after consuming edible fruit pulp, because of their vegetative means of propagation to reduce their prebearing period. For example, the tamarind seeds were in use as industrial secondary biproducts for food in some areas and so as seeds of Moringa, Moringa oleifera L.,[3].

Attempts to produce these crops with one most economically viable trait will benefit the producers, producing country and improve the industrial value with quality with ease in processing and transport of the commodity to the distant places in its fresh form and without loss or deterioration of the edible entities. For example, production of coconut fruits filled with coconut water or copra alone or coconut fruits with only fibre. Likewise, development of casew trees for production of only casew apples with a remnant nut, trees those produce only cashew nut with remnant apple will help to reduce multiscale processing costs and increase income of the farmers or producers and reduce the price of the marketted goods.

Thus tamarind is by no means the only species that could lead to economic benefit through the development of seedless fruits. The collective efforts of plant breeders and tissue culture experts may lay the path for improvement of many perennial fruit crops which are benefitting human health and nutrition. Methods to develop and maintain seedless forms of table fruit crops would im- 
prove ease of consumption of fresh fruit, may lead to increased consumption of fresh fruits, eliminate the need to separate seeds from fruit pulp during processing, and thus reduce waste. This of course would be economically advantageous for fruit processing industries of avacado (Persea americana, Lauraceae), cherry (Prunus avium, Rosaceae), coconut, guava, jack fruit, litchi (Litchi Chinensis L., Sapindaceae), loquat (Eriobotrya japonica L., Rosaceae) mango, java plum, tamarind, toddy palm, and date palm. Seedlessness could also lead to improved quality of processed fruits. Furthermore, plants bearing seedless fruits can divert resources that would otherwise go into seed formation and development into fruits with superior traits.

All these crops are having role in health and nutrition. Seedlessness not only benefits these crops, consumers and researchers but also avoids unnecessary expenses during international transport, security/quarantines, biproduct wastage, and protects native germplasm of any country producing those seeded fruits or fruit products. For this review we chose tamarind fruit crop, being a widely preferred culinery, food and pharmaceutical industries.

Therefore, the objectives of imparting seedlessness in tamarind are to increase the efficiency of production of tamarind fruit and products made from it, and to study its potential for wider adaptation as an ornamental tree in warmer climates. Furthermore, strategies for imparting seedlessness in tamarind might also be applicable to do so in other fruits as well.

\section{Genetic background of tamarind}

The chromosomal number of genus Tamarindus is 24 (2n,diploid). Very limited work has been done at molecular level to understand the genes controlling sterility and fertility restoration or seedlessness. The reasons might be its vegetative means of cultivation and mainly for fruits. In order to explore strategies for genetic improvement in tamarind, it is imperative to elucidate how various genes controlling important traits. Thus, strategies for elucidation include cytogenetics, genomics and bioinformatics as described below.

Visual descriptors of germplasm with traits useful for development of seedless cultivars in tamarind

There are more than 50 sweet tamarind cultivars under cultivation that were selected from 1811 germplasm accessions collected during 1986-87 in Thailand. They include Muen Chong, Sri Tong, Nam Pleung, Jae Hom, Kun Sun, Kru Sen, Nazi Zad and Sri Chompoo. A cultivar, Makham Waa is most popular and commonly cultivated by Thai farmers [5]. Three accessions of Thailand were selected for industrial use, one (No: 86-2-23-017) with the highest content of tartaric acid for commercial extraction of tartaric acid, the other one (No: 86-13-008) had a sugar content of 39.9\% useful for fructose production and the third one (No: 87-2-01-035) was identified with the highest concentrated tamarind flesh suitable for processing into sauces, drinks and confectionery [6].
In Philippines, 5 sweet tamarind cultivars namely Manial Sweet, Cavite, Batangas, Bulacan, and Laguna were selected from 46 collections. Author did not specify whether these collections are accessions or improved genotypes from their original collections from various locations. In India. clonal selections like PKM-1, Urigam, Prathisthan, drought tolerant cultivars like 'Yogeshwari', 'PKM', 'T3' and 'TIB' were identified as promising pulp producing tamarind cultivars out of 12 lines identified for cultivation purposes. Additionally, early, mid and late flowering types were identified through mutation by Usha in 1990. There were two types of cultivars (sweet and sour) reported from Uganda and Kenya. There was only one single collection each reported from Honduras and Burkina Faso [5].

Only 45 percent of trees are cultivated for tamarind fruits and for pulp production and remaining 55 percent included either on road or river side and unknown category [3]. These 45 percent commercially cultivated lines and 2000 genotypes [5] from published literature would help to select the high pulp yielding fruit cultivars and improve those selections for seedlessness.

Based on the literature search, it is clearly understood that tamarind germplasm was avaialable with huge variability in tree, flower and fruit traits. To date, no focussed attempts made to develop morphological description of economically important flower and fruit traits for the most cultivated species and those variations leading to parthenocarpy or seedlessness during flower and fruit development. Visual descriptors are the recorded data on all phenotypic morphological plant traits of every collection made within a crop species by a plant explorer or plant breeder or germplasm conservator at a government owned plant science research organization in any country. Therefore, it is an inevitable step to collect the tamarind germplasm and develop descriptive identifications of fruit, pulp and flower traits for each line or cultivar by using funds of central or state awarded projects.

Simultaneously, screening of existing established orchards is needed to identify any natural mutant genotype producing seedless fruits or fruits with aborted seeds, naturally induced parthenocarpy or seedlessness, or any sterile, nonfruiting lines. These important morphological traits for which genetic markers could be developed using sequencing of seedless cultivars including those developed from somatic cell hybrids and through marker technology will aid in the tamarind crop improvement for seedlessness. A visual descriptor should provide images of the plant parts phenotypically, vegetative and reproductive morphology of tree with the descriptive taxonomy, anatomy and physiological information of root, stem, leaf, flower, fruit, and their organelles, branching pattern and crown.

With the help of standard descriptors for other major fruit crops, it is easy to develop a descriptor for any collection made. 
To date, there is only one descriptor resource available digitally, if there are any offline resources at remote research locations in scientific records for some tamarind lines, immediate retrieval is required. Furthermore, conducting flow cytometric analysis on any mutant genotypes identified would be essential for future genomic research.

The morphological, molecular and biochemical descriptors with marker information of each and every trait, at every research location is a viable need in any Department of Agriculture. This helps to protect and conserve the local germplasms in their place of origin well protected in safe and deteriorated condition, and to preserve genotypes in their true to type stage at all levels of local, state and international germplasm repositories. At local county and state level research units, facilities for both electronic (digital) and descriptors of phenotypic data sheets with descriptions are foundations for ongoing as well as future research use in plant science besides appropriate refrigerated conservation units for seeds and plant propagules of various germplasm accessions.

Re-analysis of nuclear DNA content and ploidy of tamarind germplasm:(Why reanalysis? As opposed to what previous analysis?)

Re-analysis is required to know the ploidy variations in the existing tamarind lines, genetic information on chromosomes and gene loci associated with fruit traits. Advanced techniques of flow cytometry and microscopic (scanning or tangential electron microscope) analysis will allow identification of the ploidy status (mono or poly) of genotypes and cultivars available by analyzing the tissues of various plant samples such as leaves, fruits, flowers, anthers, stem and roots [7]. Through mutation or invitro culturing of haploid cells from anther (microspore mother cells, pollen) cultivars with monoploidy could be developed. These, in turn, could be used in a structured breeding program to produce improved cultivars with seedless fruits or ornamental values (Figure 1 and 2).

Identify the gene regions or genes coding for sterility, selfincompatibility, seedlessness, uniform flowering and other economic traits of importance

The first step in developing seedlessness in tamarind is to sequence DNA from various plant parts of tamarind to generate genomic information on the regions of similarity with other legume crop species . Information can also be compared among lines of tamarind germplasm available.

Information on male sterile or self-incompatable lines of tamarind is lacking. There were few germplasm resources in India, Pakisthan, Africa, Central America and France $[1,3,5,8]$. Collection of germplasm is the primary need in this crop, with continuous selection for targeted traits.

The second step is to screen this germplasm for the degree of female fertility (by counting number of seeds set per fruit) and de- gree of self-incompatibility [9] or male sterility. Cytological studies could be conducted to measure the rate of pollen tube growth that includes numerical data on reduction in growth of pollen tube or inhibition of polar nuclei in pollen grain to fertilize the egg cell nucleus in ovary of female gametophyte and counting the number of pollen germinated on stigmatic surface, number of pollen tubes emerged, number of those pollen tubes reached upto stylar region or ovary as well as the number of surviving zygotes after pollination and fertilization.

During second step, accumulation of potential genes for pod filling (pulp accumulation as well as seed development), number, color and size of flower per raceme/panicle, number of petals per flower, rate and time of flower opening, number of anthers per flower, height of the tree, number of leaflets per pinnae must be evaluated. The selection of genotypes with desirable qualitative and quantitative traits based on measurable phenotypic, biochemical, cytological and genomic sequence data can be correlated with genotypic information to choose the actual potential genotypes for further improvement and induction of sterility or seedlessness.

\section{Induction of seedlessness}

Degree of female fertility and sterility can be estimated from seeds derived by open pollination. The difference between the average number of seeds per fruit by hand pollination and that by open pollination was greater in male sterile cultivars and in selfincompatible cultivars than it was in self-compatible cultivars. Further, in open pollination, self-compatible cultivars produced very few seedless fruits while ms and SI cultivars produced many seedless fruits. These results indicated the effectiveness of ms and SI cultivars in reduction of seediness in citrus [9], as well as the effectiveness of triploidy, gametophytic SI and irradiation with soft $\mathrm{x}$-rays for induction of seedlessness in watermelon [10]. Female or male fertility and sterility varies with cultivars, age and time of anthesis, and levels of air pollution, temperature and irradiation during flowering and fruit set. Five cost-effective methods to obtain seedless cultivars from selected cultivars or improved lines in tamarind are described below.

\section{CMS gene transfer from related species for seedlessness}

There are two distinct methods to impart cytoplasmically controlled (mitochondrial gene) genetic male-sterility in tamarind lines. One method is to chose related crop species or seedless natural mutant of tamarind for cross breeding with high yielding good quality sweet tamarind cultivars through tissues culture. The cybrids produced (please see the procedure described for cybrids in the later sections) will be raised in greenhouse to observe and select those lines producing seedless fruits (from transfer of CMS gene from related male sterile crops species or seedless natural mutant of tamarind). The second method is to create mutation to alter genome structure to result in required sterility or fertility for seedlessness in tamarind fruits. Mutagens generally induce a high frequency of chromosomal changes, meiotic and mitotic irregularities. 
There are several legume crops identified with cytoplasmic male steriltiy including pigeonpea [Cajanus cajan (L.) Millsp.] [11]. Mitochondrial genome sequencing of four Cajanus genotypes revealed CMS-associated chimeric open reading frames (ORFs) that were resulted due to the mitochondrial gene rearrangements. These gene regions would be useful for production of stable F1 hybrids created by crossing highly self-pollinated lines of pigeonpea.

A similar approach can be adopted in tamarind lines from those genotypes identified with naturally induced $\mathrm{ms}$, if avaliable. Although a literature search revealed no mention of seedlessness in tamarind todate, tamarind trees have been observed in the coastal regions of Andhra Pradesh in India with seedless fruits as well as aborted seeds inside some mature pods. This suggests the possibility of natural mutation leading to either SI or ms or parthenocarpy, which needs to be experimentally confirmed. It would be important to conserve these lines for tamarind germplasm enhancement.

Ethidium bromide can be used to induce CMS and to cause changes in DNA of extranuclear (cytoplasmic) organelles like plastids and mitochondria. Acridines, streptomycin, dyes, heavy metal salts, EMS, and radiation treatments may also be used. The frequency and type of mutation recovered can vary with the dose and rate at which the mutagen is applied, the plant part to be treated (seed, pollen, vegetative propagule or complete plant), the species, the genotype within a species, oxygen level, water content, temperature, pretreatment conditioning, treatment conditions, and post-treatment conditions.

For any of the mutagenic treatments employed in an attempt to induce sterility, $\mathrm{LD}_{50}$ value must be determined as part of the protocol. Furthermore, lines with seeded fruits could be simultaneously maintained for source material. The optimum dose for inducing mutations is close to LD50 value, which varies with crop (for example, 46 Krads for Vicia faba). Mutation breeding was successful in developing cultivars of legumes with various economic traits using gamma rays in chickpea, mungbean and lentil, methyl methane sulphonate (MMS) in blackgram, and Dimethyl sulphonate (DMS) in cowpea $[12,13]$.

Sterility can be estimated by observations of floral structures during flowering, inhibited pollen tube growth, inhibition in fertilisation, inhibition of growth of fertilized ovule after double fertilisation and triple fusion to develop normal seeds, or based on the numerical count of normal and aborted seed yield starting from M1 (mutant population of first generation) and the maintenance of both male sterile and fertile lines (fertility resoter lines) by early progeny testing through bulk selection procedure as described below for six generations [14].

The planting population in M1 generation is large $(>25,000$ plants), but depends on the mutagen frequency. If there is high fre- quency, lower population size is recommended. M1 plants should not be allowed to cross pollinate, and must be planted away from any other genotypes of the same species by spacial and mechanical isolation.

Only dominant mutations are selected on each plant, selfed and harvested to raise $M_{2}$ generation. In $M_{2}$, oligogenic mutants with distinct features while in $\mathrm{M}_{3}$, polygenic traits of importance are identified and selected. However, phenotypic selection always depends on the criteria chosen for selection. In this case, selection is based on superior fruit type, pulp color and quality, seedlessness, plan theight, branching pattern, foliage, profuse flowering and cold hardiness. Physical and mechanical methods of evaluation are used for seed or fruit size, shape, weight, and density. Always made sure that desirable mutant allele found in selections is not present in the original germplasm which confirms our investigation is true through molecular genetic marker and sequencing technology approaches.

- $\quad \mathbf{M}_{\mathbf{1}}$ : Mutagen treated plants space planted (the treated seeds from one single pod will be planted in one separate row or line away from the untreated plants); Seeds from individual plants harvested to raise individual plant progenies in $\mathrm{M}_{2}$.

- $\quad \mathbf{M}_{2}$ : Individual plant progenies are grown (pod to row and tree to plot); fertile vigorous normal looking plants harvested separately.

- $\mathbf{M}_{3}$ : Individual plant progenies from selected plants grown; superior plants selected from superior progenies showing seggregation; homogenous mutant progenies may be harvested in bulk.

- $\quad \mathbf{M}_{4}$ : Individual plant progenies from selected plants grown; superior homogeneous lines harvested in bulk.

- $\quad \mathbf{M}_{5}$ : Preliminary yield trials with suitable check cultivar; superior lines selected.

- $\quad \mathbf{M}_{6}$ to $\mathbf{M}_{8}$ : Multilocation trials for yield and other attributes of importance to the breeder; outstanding lines released as cultivars.

- $\quad \mathbf{M}_{\mathbf{9}}$ : Seed multiplication for distribution. These seeds are produced for ornamental phenotype, other important economic traits needed at the time of breeding including seeds from restore lines for crossing with male sterile lines for hybrid seed production. The cuttings, graftings or other vegetative means of propagation from male steriles seedless fruit cultivars can also be multiplied and distributed. Here, seed means any material that provides next generation of seedless fruits or ornamental phenotype for our review.

\section{Production of haploids by pollen culture}

Anther or pollen culture is a proven technique for generation of haploids in field crops for hybrid seed production. In crops where 
both vegetative propagation and propagation from seed are possible, induced autoploidy is the best option [14]. Therefore, it can be applied in tamarind where seedlessness in fruits is the research target to benefit small scale tamarind fruit processing as well as the largest established pulp industries. Thirty-six genotypes classified for different fruit pulp types [15], is a good resource population to study and employ anther culture technique in tamarind for production of haploids or generation of seedless fruit cultivars.

Pollen grains are sticky, dimorphic (40-42 $\mu \mathrm{m}$ and $22-25 \mu \mathrm{m})$, radially symmetrical, spheroidal and had very low levels of sterility $(<2 \%)$. However, fruit set was also observed to be low $(1-2 \%)$ under natural conditions, which means only 1-2 flowers set fruit out of 100 flowers. Use of insect vectors resulted in increased for fruit set (70-80\%) under controlled conditions. This observation indicates the self-incompatibility nature of tamarind species $[5,16]$. The self-incompatible lines could be identified from available 2000 genotypes, an approximate number obtained from literature published from different tamarind growing regions of the globe.

Production of cybrids by somatic cell fusion (protoplast fusion)

The unique concept of cytoplasmic organelle control of male sterility is useful in production of seedless citrus cybrids [17]. Diploid and tetraploid plants were generated through interspecific fusion combinations of embryogenic suspension-derived protoplasts of male-sterile Satsusma mandarin (Citrus unshiu Marc.cv. Guoqing No.1 or G1) as the callus parent and leaf protoplasts of other seeded cultivars as leaf parents by targeted transfer of CMS. The determination of genetic identities of regenerated progeny were sensitive and highly technology driven, due to flow cytometric analysis at molecular level. Here, genetic identities means analysis of CMS genes specific to seedlessness or to different ploidy to confirm the tansfer of CMS genes from donor parent to the fused cybrids formed from protoplasts of both parents. This kind of protoplast fusion is applicable to woodey plant species [18]. Thus, it is a potential tool for development of tamarind population with various ploidy levels for seedless cybrid fruit production.

For tamarind crop, the species identified with cytoplasmic male sterility can be obtained from either sub-family Fabacease or Cesalpinaceae of family Leguminosae. There are several crop species identified with CMS and the CMS associated ORFs with potential gene regions specific to male sterility, which could be useful for genetic transformations for cultivar improvement. These species include pigeonpea and chickpea. The steps to standardize the method of protoplast fusion for tamarind cybrids from leaves or embryoids and for generation of polyploids or for transgenics from related crops species is detailed below, based on the somatic hybridization technique [18-20].

Step 1

Selection of parents (one parent with CMS and or seedless type fruit cultivar and the other parent with seeded fruits selected from those of various fruit shapes and size).

\section{Step 2}

Isolate protoplasts from both parent types, using mechanical means using plastic tape or razor blade if possible, from fully expanded leaves of low starch content from greenhouse grown plants under high humidity, low light conditions. For tamarind, use desalted enzymes like cellulases, macerases or pectolyases to enhance protoplast isolation and to replace mechanical means due to its smallest leaflet size. Determine enzyme quality and quantity, time of exposure, and the concentration of osmoticum empirically.

Isolation can be done using cut leaf pieces with a 95 per cent recovery when extracted in a liquid sterile water medium with 1.5 per cent cellulase at low speed shaking using an orbital shaker at 25-50 rpm for $4.5 \mathrm{hr}$ at room temperature. With the use of microscope, isolate protoplasts and transfer to culturing medium in a test tube or sub-culturing tray with multiple wells or multi-well plate to raise regenerated cybrids from multiple parental combinations after step 3. Here, one pair of protoplasts from each parental combination is allowed to grow in a specified well of the multi-well plate or tray.

Step 3

Fusion of protoplasts for somatic hybridization of both parental types. Use fusogens (fusion inducing agents) if the natural fusion of protoplasts is poor. Relatively shorter exposure times to chemical like polyethylene glycol (28-50\% PEG 1500mw) for adhesion and fusion of protoplasts as well as use of fusogens like 5.5 per cent sodium nitrate, were the most common practices for enhanced quality of intergeneric or interspecific protoplast fusion.

Use of mannitol and sucrose at various concentrations during incubation of protoplast mixture at $35 \mathrm{oC}$ for $5 \mathrm{~min}$ followed by centrifugation at $200 \mathrm{~g}$ for $5 \mathrm{~min}$ as well as use of small sized protoplasts were the most important factors for viability of the protoplasts and for the highest fusion percentages. In some cases, the protoplast mixture is exposed to high $\mathrm{pH}$ (10.2) and a high calcium concentration (50mmol/1) for 30-35 $\mathrm{min}$.

\section{Step 4}

Fused protoplasts (heterokaryon) can be identified with green florescent protein or chlorophyll florescence by their morphology and the number of chloroplasts from leaf derived protoplasts can be counted. After cell wall formation, division of fused cells occurs quickly if maintained sterile culture media. Changing the culture medium at weekly intervals to maintain the $\mathrm{pH}$, tight sealing of the fusion culture to avoid desiccation, maintaining high humidity, diluting culture media to provide aeration and changing liquid medium to the solid medium are recommended to improve the efficiency of regenration.

\section{Step 5}

The cybrids will be identified as seedless or transgenic CMS lines with ORFs, based on morphological and molecular means using genomic marker technology. The cybrids will be moved to temperature and humidity controlled growth houses, green houses, later to research farms for studies on fruit and yield attributes of cybrid lines and finally to field for commerical planting after multiple, multilocation yield evaluation trials. 
Use of growth inhibitors during pre-anthesis stage:

$\mathrm{GA}_{3}$ is well known growth regulator to induce parthenocarpy and seedlessness in fruit crops. Seedlessness was attained with an optimum GA3 concentration of $0.15 \mathrm{mM}$ when applied during pre and post anthesis stages on field grown high quality seeded table grape culitvar "Swenson Red" both by dip and spray applications [21]. Though application of growth regulators is a common cultural practice in tamarind orchards for year-round flowering and fruit set, no attempts to use growth regulators to induce seedlessness are known.

Chemicaly induced seedlessness introduced by Kihara and Nishiyama in 1939 can be costly, such as the application of colchicine to produce triploid watermelon. Triploidy in watermelon imparts seedlessness in fruits, but seeds were costly for farmers. Thus, in other crops, other methods of new cultivar development were more successful in watermelon for long term use by farmers [22].

Research experimentation conducted in climate controlled greenhouse enhance fruit-set compared to field cultivation, due to short stigmatic receptivity period of 48 hours during anthesis [16]. The market value of fresh and sweet tamarind fruits could be enhanced by the development of seedlessness. This could be achieved by application of growth regulators under protected cultivation (either high tunnel or greenhouse) at bud stage or pre- or post-anthesis stage after open or cross pollination in a way to arrest the growth of fertilised ovule inside an embryo. Initial steps would include screening for the growth regulator and its optimum concentration to induce seedlessness followed by identification of the optimum stage to retrieve maximum number of seedless locules per fruit and seedless fruits per cluster in a single tree.

Collection of pods from a single parent tree observed with seedlessness

There are trees producing both seeded and seedless fruits due to natural mutation occurring during pod development or during pollination [23]. Identifying such lines from already established germplasm orchards is the first step. Following which collection of pods with at least one aborted seed inside a single pod, where remaining seeds will help to raise the population of first generation. This approach will help in securing expected progenies with improved number of aborted seeds per pod due to natural methods of identifying sterile, seedless, naturally induced parthenocarpy or mutation. Development of pure mutant lines with seedlesness in fruits takes five to six generations of selection.

Each hermaphroditic flower in tamarind contains three stamens and a pistil with upto 14 ovules. Open flowers with indehiscent (closed) anthers had no pollen tube growth while open flowers with dehiscent anthers had pollen tube growth reaching ovules. A greater number of pollen tubes, their growth due to open pollination and hand cross pollination were observed compared to hand self-pollination. However, not all pollen resulted in fertilization due to callose formation or some unknown incompatibity reaction between pollen and ovule [24]. This was identified as prezygotic self-incompatibility controlled by multiple alleles at the gene loci, and has potential for research to develop seedless fruits in tamarind. There was no observed post-zygotic self-incompatibility, but self-pollinated flowers produced a high percentage of aborted seed in fruits compared to cross pollinated flowers.

Through gametophytic self-incompatibility, the good quality and sweet seedless watermelons were produced successfully only by irradiating pollen from male flowers of diploids with $600 \mathrm{~Gy}$ soft $x$-rays and then fertilizing female flowers with the irradiated pollen [10]. A similar technique can be employed in tamarind. After soft x-ray irradiation of pollen and artificial pollination with this pollen will result in abortion of the embryo, inhibition of embryotic development causing degeneration of endosperm leading to production of seedless fruits. It is the cheapest and safe technique compared to previously described techniques, though it requires scanning electron microscopy (SEM) for observation of embryo sacs. It has been found successful in that soft x-rays did not affect the viability of the pollen and resulted in embryo formation after double fertilization, but the growth of the embryo was arrested due to damage to the pollen DNA. Thus, ovules inside the degenerating embryos did not develop into normal seeds.

Maintenance of tamarind lines developed for seedless $F_{1}$ hybrid fruits

The following steps must be kept in mind in every generation for obtaining a pure breeding population.

1. Morphological screening of germplasm for qualitative and quantitative traits and fruits related to seedless fruits or naturally induced parthenocarpy in field grown trees collected from various locations after introduction.

2. Development of complex or three way or diallel mating designs depending on the traits chosen for improvement especially pod traits (thin and smooth rind surface, heavy mesocarp region with pulp, small seed size, short and wide pods), flower color, number of flowers, leaf quality, branching type, plant height etc., The newly improved traits of new cultivar should also be included in the "visual descriptors" of tamarind cultivars immedietely upon development.

3. Induction of seedlessness by using growth regulators and by changing ploidy by colchicine to develop polyploids for use in plant breeding to develop triploids and tetraploids as well as anther culture for haploids in tamarind.

The maintenance of $F_{1}$ population is similar to the procedure adopted for other field legume crops. Restorer lines, growth regulators and tissue culture techniques have been used to maintain the sterile, triploid populations of several fruit crops. Because 
tamarind is prone to high cross pollination due to its protogynous flower structure, it is always beneficial to grow the population of seedless cultivars away from the original seeded cultivars to avoid cross contamination of seeded types from seedless fruit types. Flow chart shows the various strategies to develop tamarind with seedless fruits (Figure 1)

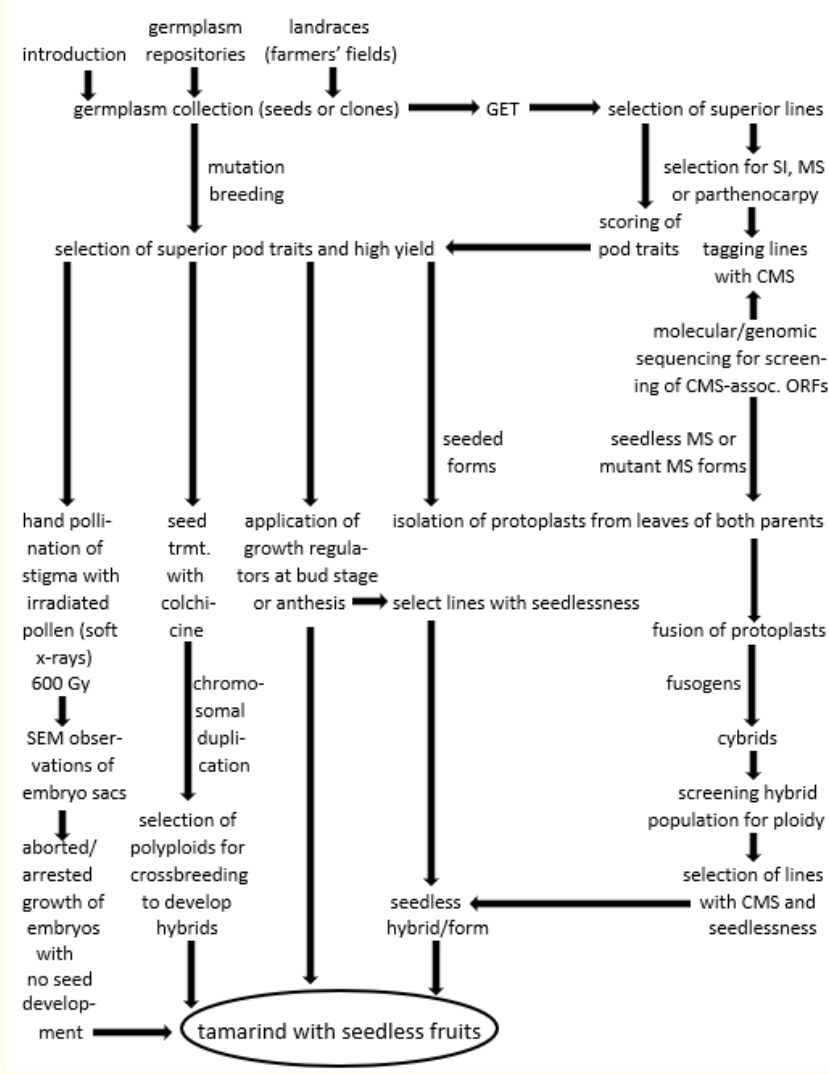

Figure 1: Strategies for crop improvement of tamarind for seedless fruits.

GET: Germplasm Evaluation Trial; SI: Self Incompatability; CMS Cytoplasmic Male Sterility; MS: Male Sterility; ORF: Open Reading Frames.

Germplasm with broad genetic diversity for the fruit traits will be collected from land races, by germplasm exchange or from primary gene pool of germplasm resource centers. The morphological and agronomic trait scoring should be conducted for pod traits like number of locules, pod size and weight, maximum and minimum number of seeds per pod, timing of pre- and post-anthesis, fruit set and length of time required for pod formation and ripening. Selection of lines for high yield, maximum pod size and weight, optimum number of locules is important. Identification of any cultivars with naturally induced seedless fruit type, any lines with ms or SI during mutant population studies, and screening of these lines selected for molecular similarities for genomic regions of CMS and for associated mitochondrial gene ORFs should be conducted. This is done by sequencing DNA of selected lines with desirable fruit quality and that induce male sterility. Development of maintainer and restorer lines simultaneously during breeding program is as important as identification of male sterile and self incompatibile lines in tamarind. Following the six - to eight- year breeding program using CMS lines or by using transgenics from related genera to develop cybrids with seedless fruits is possible in tamarind.

\section{Tamarind crop for ornamental use}

The best ornamental traits of the tamarind tree include 1) dense foliage (greenish purple when immature) with beautiful compound leaves with glossy/waxy upper surface to reduce evapotranspiration water loss 2) sympodial branching pattern on matured trees, creating a beautiful specimen tree of a garden with a large crown or, in numbers, a protective hedge row or border planting 3) Beautiful pink, yellow or white flower panicles, 3) adaptability to a wide range of soils including those with sandy or sandy loam soils with low water holding capacity and low fertility 4) high response to pruning, grafting and other vegetative propagation techniques with more than 80 percent success in graft-take, 5) Retention (lack abscession) of ripened fruits and leaves on tree, although leaf drop can occur during drought.

\section{Induction of profuse flowering and dwarfness}

Mutation breeding is used most frequently to induce modifications more than the spontaneous rate of 10-6 for an individual gene and can be applied to screen for specific traits related to plant size habit, foliage and flowering. Treatment of seeds or young seedlings with mutagens followed by evaluation and selection will result in improved forms of mutant, dwarf phenotypes with foliage and attractive flowers with aesthetic features. The protocol includes selection of mutant lines with profuse flowering and dwarfness in every generation followed by progeny testing to obtain superior ornamental phenotypes [14] (Figure 2).

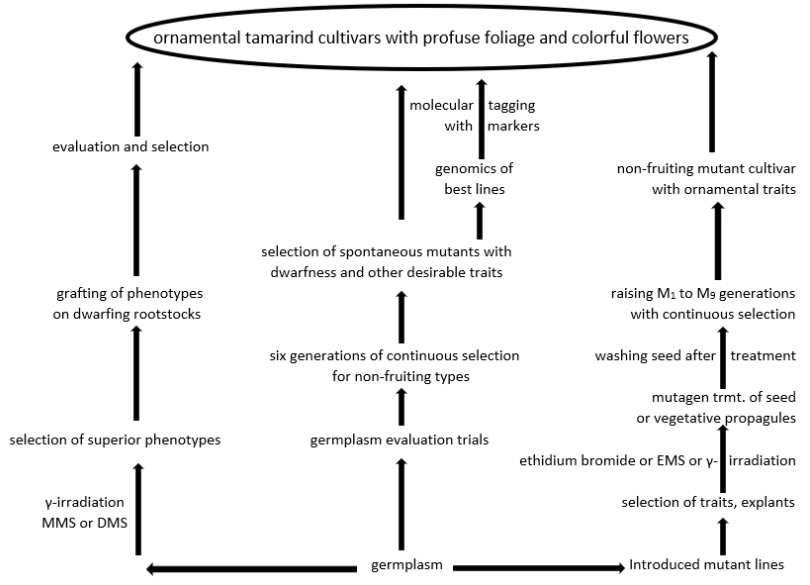

Figure 2: Strategies for crop improvement of tamarind for ornamental use.

DMS: Dimethyl Sulphonate; MMS: Methyl Methane Sulphonate; EMS: Ethyle Methyle Sulphonate; M1 to M9: First to Nine Generations of Mutant Population; $\gamma$ : Gamma.

Alternatively, grafting and other vegetative means can be used to propagate desirable selections with various flowering types [1] and various fruit shapes, colors $[3,5]$. The fruit pulp colors are red 
and brown, while flower colors are white, yellow, pink and red. More than 15 variants in fruit and seed size and shape, although no variation observed for rind or shell color.

Measurable variations in floral traits, including inflorescence length (4-6cm), inflorescence per branch (8-16), flowers per inflorescence (8-17), flowers per branch (101-280), style length (4$5 \mathrm{~mm}$ ) and ovary length (6-7mm) in clonal selections NBN, RDB and JRK were reported [5]. They also reported that flowering in tamarind was commonly seen in spring or summer, that longer vegetative terminal shoots produced more flowers, and that foliage production was highly correlated with terminal shoot length, a useful character to evalaute genotypes. In general, flowering and fruiting was highly depended on local agroclimate and altitude [3].

Tamarind is a protogynous species, with flowering starting in August-September and with ripened fruits produced 8-10 months later. Ripe fruits were available from April to June in Florida, Central America and West Indies while in Southeast Asian countries, Philippines ripened fruits were available in August to October [5]. These findings convey that the flowering in tamarind is strictly controlled by the growing environment and the tree has ability to flower and fruit throughout the year. Anther dehiscence occurs during 8-10 hrs in the morning and the stigmas are receptive for 48 hours with peak receptivity on the day of anthesis [16].

The following phenological stages of flowering are appropriate to observe the degree of flower retention versus flower drop: (flower bud, elongated flower and open flower with indehiscent anthers). Observations would allow selection of forms with longer periods of flowering. Further studies are required to know the genetic factors controlling early abscission or flower drop.

Production of essential oils from flowers

Tamarind is not explored for essential oil extraction from flowers, leaves, seeds or fruits. Because, I did not get literature as of September 2019. The selection of lines from the presented strategies of breeding programs would be useful.

\section{Conclusion}

Use of transgenics, tissue culture and other technological methods are useful to develop improved cultivars with seedless fruits, and more desirable, ornamental traits. Cybridization and artificial fertilization through irradiated pollen are recommended to induce haploidy, in turn, produce trees with seedless fruits. Germplasm screening at the molecular level and mutation breeding are essential for selection of lines for ornamental use. The great range of variability exists in germplasm related to vegetative and reproductive traits holds great potential for genetic improvement of the species.

Gene sequencing of plant samples at various stages of fruit growth and development will reveal more information on those genes upregulated after fruit set, during fruit growth and those genes down regulated after fertilisation and during seed development in parthenocarpic or seedless fruits of tamarind F1 hybrids. Similarly, identification of genes related to floral organ development may ultimately result in improved flowering traits for ornamental use.

Finally, establishment of a small-scale germplasm conservatory unit for tamarind and other tropical crops is needed. Such a resource, with morphological, molecular and biochemical descriptors with marker information for tamarind would protect and conserve existing germplasm in a safe and undeteriorated condition Future collaborations will be enhanced through the sharing of germplasm and additional findings related to gene regulation and breeding techniques for tamarind.

\section{Acknowledgement}

Authors like to thank Mr.Pradeep Kumar Sripathi, IT professional for providing computer and highspeed internet facility to search available literature and successfully complete this mini-review on time. Authors like to acknowledge Dr. Leonardo and Dr. Taghavi for providing digitally unaccessable two good research articles immediately upon request in support of this peer review publication.

\section{Bibliography}

1. Usha K. "Studies on dynamics of vegetative and reproductive growth in tamarind (Tamarindus indica L.)", a PhD thesis submitted for the award of degree "PhD in Horticulture" to Univ. Agric. Sci., Bangalore (1990).

2. Narina SS and Catanzaro CJ. “Tamarind (Tamarindus indica L.): An underutilized fruit crop with potential nutritional value for cultivation in the United States of America: A Review". Asian Food Science Journal 5 (2018): 1-15.

3. Narina SS., et al. Book Chapter 44: Moringa and Tamarind-Potential drought tolerant perennial crops with nutritional values. In: Pessarakli, M (ed) Handbook of Plant Crops and Stress. CRC Press, Taylor and Francis Group, Boca Raton, Florida, USA (2019): 817-835.

4. Maninder Kaur and Sukriti Singh. "Physicochemical, Morphological, Pasting, and Rheological Properties of Tamarind (Tamarindus indica L.) Kernel Starch". International Journal of Food Properties 19 (2016): 2432-2442.

5. El-Siddig K., et al. "Book on Tropical fruit trees "Tamarind (Tamarindus indica L.)” Edited by Williams JT., Smith RW., Haq N., and Dunsiger. Published in 2000 by International centre for underutilized crops, University of Southhampton, Southhampton, S017 1 BJ, UK Revised in 2006 by Southhampton centre for underutilized crops, Southhampton, UK (2006).

6. Feungchan S., et al. "Tamarind (Tamarindus indica L.) Plant genetic resources in Thailand". Thai Journal of Agricultural Science (1996a): 1-11.

7. Galbraith WD., et al. "Analysis of Nuclear DNA content and ploidy in higher plants". Current Protocols in Cytometry (1997). 
8. Diallo BO., et al. "Genetic diversity of Tamarindus indica L. populations: Any clues on the origin from its current distribution?" African Journal of Biotechnology 6 (2007): 853-860.

9. Yamamoto M., et al. "Relationship between sterility and seedlessness in citrus". Journal of the Japanese Society for Horticultural 64 (1995): 23-29.

10. Qu H-y., et al. "The mechanism of seedlessness in watermelon generated using soft-x-ray irradiated pollen". African Journal of Agricultural Research 11 (2016): 2200-2204.

11. Tuteja R., et al. "Cytoplasmic Male Sterility-associated chimeric open reading frames identified by mitochondrial genome sequencing of four Cajanus genotypes". DNA Research 20 (2013): 485-495.

12. Kharkwal MC., et al. "Induced mutations for improvement of chick-pea, lentil, pea and cowpea. International Atomic Energy Agency (IAEA): IAEA. Joint FAO/IAEA Div. of Isotope and Radiation Applications of Atomic Energy for Food and Agricultural Development, Vienna (Austria); Panel proceedings series (1988): 89-109.

13. Kumar S., et al. "Mutation breeding in Chickpea". Advances in Plant and Agriculture Research 9 (2019): 355-362.

14. Walter Fehr. "Principles of Cultivar Development: Theory and Techniques". Agronomy Books 1 (1991): 551.

15. Algabal AQAY., et al. "Estimation of genetic variability in tamarind (Tamarindus indica L.) using RAPD markers". International Journal of Plant Breeding and Genetics 5 (2011): 10-16.

16. Nagarajan B., et al. "Phenology and controlled pollination studies in Tamarind". Silvae Genetica 47 (1997): 237-241.

17. Guo WW., et al. "Targeted cybirdization in citrus: Transfer of Satsuma cytoplasm to seedy cultivars for potential seedlessness". Plant Cell Report 22 (2004): 752-758.

18. Jude W Grosser. "Observations and suggestions for improving somatic hybridization by plant protoplast isolation, fusion and culture". Horticultural Science 29 (1994): 1241-1243.

19. Olivares-Fuster O., et al. "Green fluorescent protein as a visual marker in somatic hybridization". Annals of Botany 89 (2002): 491-497.

20. Gosch G., et al. "Isolation, culture, and fusion studies on protoplasts from different species". Journal of Protoplasma 85 (1975): 327-336

21. Fellman C., et al. "Gibberellic acid-induced seedlessness in field grown vines of "Swenson Red" Grape". Horticulture Science 26 (1991): 873-875.
22. USDA-NAL (2019). Andrus CF, Seshandri VS and PC Grimball (1971). Production of seedless watermelons. Technical bulletin 1425. USDA-National Agricultural laboratory, current serial records (2019): 16.

23. Personal communication. Author Satya Narina observed tamarind trees, made a note on occurance of natural mutation and seedless fruits besides few stoney locules in fruits (2018).

24. Diallo BO., et al. "Breeding system and pollination biology of the semidomesticated fruit tree, Tamarindus indica L. (Leguminosae: Caesalpinioideae): Implications for fruit production, selective breeding, and conservation of genetic resources". African Journal of Biotechnology 7 (2008): 4068-4075.

\section{Volume 3 Issue 11 November 2019}

(C) All rights are reserved by Satya SS Narina., et al. 